(C) 2015 IEEE. Personal use of this material is permitted. Permission from IEEE must be obtained for all other uses, in any current or future media, including reprinting/republishing this material for advertising or promotional purposes, creating new collective works, for resale or redistribution to servers or lists, or reuse of any copyrighted component of this work in other works. 


\title{
Optimized Demand Response Bidding in the Wholesale Market under Scenarios of Prices and Temperatures
}

\author{
J. P. Iria, F. J. Soares and R. J. Bessa \\ Instituto de Engenharia de Sistemas e Computadores, INESC TEC \\ Porto, Portugal \\ jpiria@inescporto.pt, fsoares@inescporto.pt and rbessa@inescporto.pt
}

\begin{abstract}
Demand aggregators are new market players that represent a group of consumers in the electricity market. This paper proposes an aggregator model responsible for gathering residential and commercial consumers, which has the role of managing their flexible consumption in the day-ahead electricity market. A methodology to optimize the aggregator's bids is also presented. It optimizes the scheduling of the flexible loads taking simultaneously into account the consumers' preferences and temporal trajectories of forecasted outdoor temperatures and electricity prices. The proposed methodology was tested using a case study with 200 residential and commercial consumers from the Iberian market.
\end{abstract}

Index Terms-Aggregator, wholesale market, demand response, probabilistic scenarios.

\section{INTRODUCTION}

The residential and commercial sectors together represent more than $50 \%$ of the electricity consumption in EU-27 [1]. The current electricity system does not include active participation of these parties (small consumers) in the electricity markets. The participation of theses consumers is acknowledged as the inevitable solution to enhance the economic efficiency of electricity markets, reduce peak demand and price volatility, and improve the reliability of electric power systems [2]-[3]. The implementation of advanced communication infrastructures and other smart grid technologies promises to increase the participation of these consumers in the electricity market.

The demand aggregation is known as an efficient solution to increase the exposure of large volumes of small consumers to the electricity market, since they do not have enough size to participate directly. In this context, the aggregator represents a solution for the participation of this type of consumers in the electricity market.

The aggregator is an intermediary between the small consumers, the electricity market, the distribution system operator (DSO), and the transmission system operator (TSO). It has the responsibility of coordinating the consumer's behavior in the market. This concept was explored by the

This work was made in the framework of the BEST CASE project ("NORTE-07-0124-FEDER-000056") financed by the North Portugal Regional Operational Programme (ON.2 - O Novo Norte), under the National Strategic Reference Framework (NSRF), through the European Regional Development Fund (ERDF), and by national funds, through the Foundation for Science and Technology (FCT). It was also financed by the FCT - Fundação para a Ciência e a Tecnologia (Portuguese Foundation for Science and Technology) within project UID/EEA/50014/2013.
European project ADDRESS [4], where the aggregator gathers domestic consumers, in order to provide (sell) active demand products to the electricity market or directly to the system operators.

The aggregator interfaces with the end users through a device called Energy Management System (EMS) [5]-[6]. The function of this local controller is to manage the electric power consumption of the flexible loads, according to the customer's preferences and aggregator's requests. Recent works about EMS have been dedicated to the management of appliances under real-time electricity pricing [7]-[8]. These studies are focused in reducing the electricity bills of the end users by shifting their energy consumption from the periods of higher prices to the periods of lower prices. However, these works do not considered a bidirectional exchange of information between a retailer (i.e. an aggregator) and consumers, since the retailer only defines or sends hourly retailing tariffs to the consumers.

The problem addressed in this paper consists of an aggregator, which manages the flexible consumption of residential and commercial consumers, under the smart grid paradigm. The aggregator, via EMS, explores the flexible load of its clients to increase its profits by minimizing the cost of the electricity purchased. Thus, the aggregator presents buying bids in the day-ahead spot market to satisfy the consumption of its clients.

To support the participation of the aggregator in the dayahead sessions of the market, we formulate a multi-temporal optimization problem. This method forecasts the optimal scheduling of the flexible loads, based on temporal trajectories of forecasted outdoor temperatures and electricity prices. This optimization is run by the EMS of each consumer. Based on these forecasts, the aggregator optimizes the buying bids to be presented in the day-ahead market.

The consideration of multiple temporal trajectories (or scenarios) hedges the risk of the aggregator, since the risk associated with the electricity prices and the thermal loads forecasting is directly modelled in the optimization problem. 


\section{AGGREGATOR FRAMEWORK}

\section{A. Aggregator Model}

The aggregator definition found in [4] was closely followed. In this paper, the aggregator was considered to be a financial entity responsible for gathering residential and commercial consumers, in order to maximize its profit in the electricity market and decrease the consumers retailing price by minimizing the cost of purchased electricity.

The aggregator buys electricity for small consumers in the electricity market based on the forecasted load for the next day. This load is divided in two types: flexible and inflexible. The inflexible load is forecasted in aggregate form by the aggregator. However, the flexible load is forecasted by the EMS of each client and after that clustered by the aggregator.

Two different groups of clients are foreseen:

- Type A) a client who allows the EMS to schedule the operation of their flexible loads, according to a temporal trajectory of forecasted electricity price transmitted by the aggregator.

- Type B) a client who does not allow the aggregator to control the scheduling of their loads. The load of these clients is considered inflexible. In this case, the aggregator is only an electricity retailer.

In order to promote the maximum participation of flexible loads in this type of scheduling, the aggregator can set special tariffs. These tariffs are cheaper than regular retailing prices and they are applicable to flexible loads. Concerning inflexible loads, they pay regular retailing prices.

The aggregator represents these clients in the electricity market and retains a profit that depends on its bidding strategy and scheduling strategy adopted for the flexible loads. The benefit for the aggregator is the possibility of increasing its profit by decreasing the costs of purchasing electricity in the market. In exchange, the aggregator can offer cheap retailing prices or a discount in the monthly electricity bill, in particular for type A clients.

\section{B. Interaction with Energy Management System (EMS)}

The aggregator interfaces with the consumer through a control device installed by it, called Energy Management System. The role of this device is to optimize and manage the electric consumption of the end user. On one hand, the EMS receives information from the aggregator and on the other hand from the end user, specifying his/her own preferences. The EMS also retains information on consumption characteristics of the loads installed at customer's site, energy price paid to the aggregator, and possibly other information related to forecasting electricity consumption.

In this framework, the EMS has two different roles. At day $\mathrm{D}$, the EMS is responsible for forecasting the consumption of the flexible loads for day D+1, taking simultaneously into account multiple temporal trajectories of forecasted outdoor temperatures and one of electricity price. The scheduling of the flexible loads also takes into account consumer's preferences. At day D+1, the EMS is responsible for managing the flexible loads, in order to minimize the deviations between the forecasted and real consumption. This functionality is not addressed in this work.

The communication between the aggregator and EMS should be bidirectional: upstream information (load profile of the flexible loads for day D+1) from the EMS to the aggregator; downstream information (multiple temporal trajectories of forecasted outdoor temperatures and one of electricity price for day $\mathrm{D}+1$ ) from the aggregator to the EMS.

The Aggregator forecasts multiple scenarios of electricity prices and transmits only one to each client, in order to avoid the scheduling of all flexible loads in the same periods. This approach allows hedging the risk of the aggregator in the bidding process by distributing the flexible load along the low price hours.

\section{Interaction with the TSO, DSO and Electricity Market}

The aggregator is an important actor in the transmission and distribution network, and market operation. The interaction between the TSO, the DSO and the aggregator is as follows:

- At the beginning of the day-ahead spot market session, the aggregator buys electrical energy for its clients.

- $\quad$ The TSO jointly with the DSO make the validation (and bid correction if necessary) of the aggregators' bids, e.g. determining consumption reduction when there are congestions in the transmission and distribution networks. Since the DSO does not have direct access to the market information, this coordinated management will require the exchange of information between the TSO and DSO, in an effective and timely manner. Therefore, it will be necessary to develop new management procedures for the DSO and change the current electricity market and regulatory rules; details about possible frameworks can be found in [4] and [9].

\section{Management Model}

At day $\mathrm{D}$, the aggregator forecasts for each hour of day D+1: the aggregated inflexible load of all its clients, the multiple temporal trajectories of forecasted outdoor temperatures and electricity prices. Based on multiple temporal trajectories of forecasted outdoor temperatures and one of electricity price, the EMS schedules the operation of the flexible loads, in time steps of 15 minutes, for day D+1. This scheduling also takes into account the client preferences. Afterwards, the aggregator gathers all forecasted consumption (flexible and inflexible), in order to define the hourly bids for buying electrical energy in the day-ahead market.

\section{PARTICIPATION OF THE AGGREGATOR IN THE DAY- AHEAD MARKET}

\section{A. General Framework of the Market}

In this paper only the day-ahead spot market is considered.This framework is based on the Iberian electricity 
market. However, the methods described in this paper can be easily adapted to different market rules.

The day-ahead spot market has a uniform price and double-side auction. The market agents may present buy and sell hourly bids that cover all $24 \mathrm{~h}$ of the next day. The aggregator is assumed to be almost a price-taker that only presents bid for energy quantities. The market gate closure occurs at $10 \mathrm{~h}$.

\section{B. Identification of Flexible Loads at Low Voltage Level}

Flexible loads are appliances/equipment's that can change their electricity consumption, according to control actions. At low voltage level, loads such as thermostatic controlled loads, electric vehicles (EV), washing machines, dishwashers and clothes dryers can be identified as flexible. In this work, refrigerators, electric water heaters $(\mathrm{EWH})$, inverter air conditioners (IAC) and EV are considered flexible. All the other loads are considered inflexible.

\section{Generation of Temporal Trajectories}

The uncertainty in multi-temporal decision-making problems should be represented by a set of temporal trajectories that respect the marginal forecasted distribution and capture the temporal dependency of forecast errors. These trajectories are generated with a three-step approach.

Firstly, point forecasts for the outdoor temperature and electrical energy price are generated. The outdoor temperature is forecasted by a Numerical Weather Prediction model, i.e. the Weather Research and Forecasting model [10]. The price forecast is produced with an additive model (see [11]) that uses past observations and forecasted wind power penetration as input variables.

Secondly, probabilistic forecasts (i.e. set of quantiles) are produced by the quantile regression method using the temperature and price point forecast as explanatory variable [12]. It is important to stress that these probabilistic forecasts do not capture the temporal dependency between forecast errors of different lead-times. The third step consists in generating temporal trajectories based on the Normal-toAnything method [13] that provide information on the development of the prediction errors through the time horizon.

The method takes as inputs the forecasted quantiles and also the observed temperature and price values. The forecast errors are made Gaussian by applying a transformation with the inverse of the Gaussian cumulative distribution function. This results in a Gaussian random variable with zero mean and unit standard deviation. Considering the vector with all the forecasts for each lead-time, it is assumed that the random vector follows a multivariate Gaussian distribution, with mean values being a vector of zeros and a covariance matrix.

The temporal interdependence structure is represented by the empirical covariance matrix. A specified number of trajectories are obtained through sampling from an inverse cumulative distribution function.
Figure 1 and 2 show the temporal trajectories of forecasted outdoor temperatures and electricity prices used in this work.

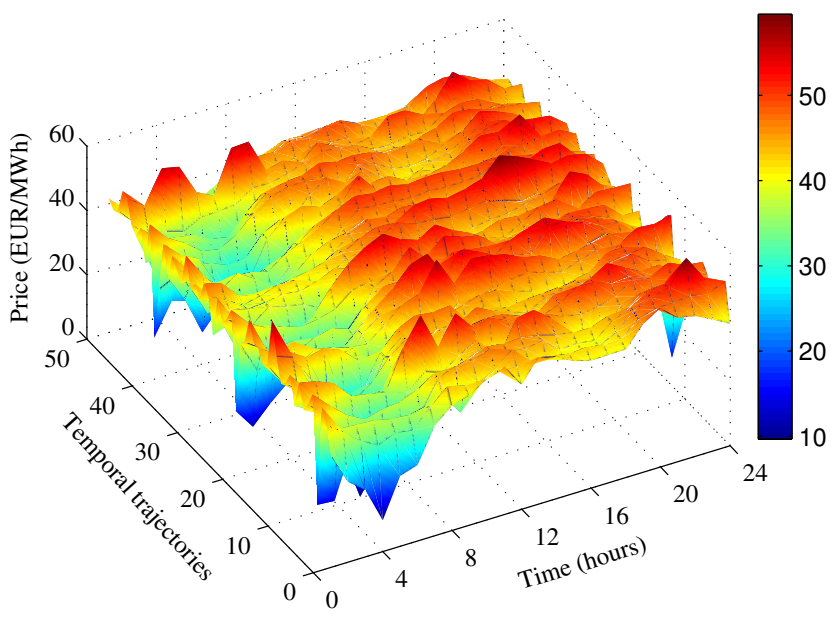

Figure 1. Fifty temporal trajectories of forecasted electricity prices for the participation of the aggregator in the Iberian Market ( $9^{\text {th }}$ August of 2010)

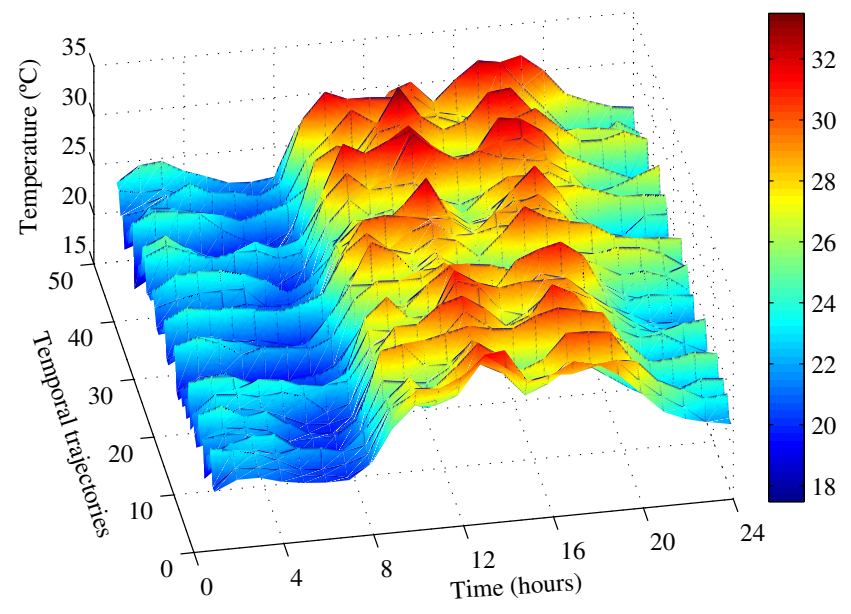

Figure 2. Fifty temporal trajectories of forecasted outdoor temperatures for August $9^{\text {th }}$ of 2010 in Porto (Portugal)

\section{Schedulling of Flexible loads}

Each type A client has an EMS that runs a multi-temporal optimization method in the beginning of day D. The function of this method is to optimize the scheduling of the flexible loads, in order to minimize the cost of purchased electricity by the aggregator in the day-head spot market. The objective is to shift the consumption of the flexible loads from the periods of high prices to the periods of low prices.

The multi-temporal optimization method is composed by different mathematical models, with the goal of optimizing the scheduling of each flexible load. The models can assume different formulations depending on the type of flexible load and temporal trajectories considered.

The optimal scheduling of the IAC for a price scenario $j$ is given by the following mathematical formulation: 


$$
\operatorname{Min} \sum_{t=1}^{N T} \text { Price }_{t, j} P_{t} \Delta t
$$

$$
\begin{aligned}
& \text { Subject to } \\
& \partial_{t} \in\{0,1\} \\
& \partial_{t} P^{\text {Min }} \leq P_{t} \leq \partial_{t} P^{\text {Max }} \\
& \theta_{i, t+1}=\theta_{i, t}-\frac{\Delta t}{C R}\left(\theta_{i, t}-\theta_{i, t}^{o}+R P_{t} \eta+w_{t}\right) \\
& \partial_{t} \theta^{\text {Min }} \leq \theta_{i, t+1} \leq \theta^{\text {Max }} \\
& t=1, \ldots, N T ; i=1, \ldots, N O
\end{aligned}
$$

The objective function (1) consists in minimizing the cost of buying electricity in the day-ahead market through setting the electric power $\left(P_{t}\right)$ consumed by the IAC in each time interval $t$. The parameters in (1) are as follows: Price $_{t, j}$ is the forecasted price of the temporal trajectory $j$ at time interval $t ; N T$ is the number of periods (e.g., 96 periods of 15 $\min ) ; \Delta t$ is the duration of the time intervals in hours.

The model has four constraints. The first constraint is (2), and guarantees that the IAC has two modes of operation $\left(\partial_{t}\right)$, $\mathrm{ON}$ and OFF. The second constraint is (3), and ensures that the electric power of the IAC does not exceed its technical limits $\left(P^{\operatorname{Min}}-P^{\operatorname{Max}}\right)$, when it is $\mathrm{ON}$. The third constraint is (4), and is a physically-based load equation [5]-[6] that sets the temperature inside the room $\left(\theta_{i, t}\right)$, for all trajectories of forecasted outdoor temperatures $i\left(\theta_{i, t}^{o}\right)$. The parameters in (4) are as follows: $C$ and $R$ are the thermal capacitance and thermal resistance of the room; $\eta$ is the coefficient of performance; $N O$ is the number of temporal trajectories of forecasted outdoor temperatures; $w_{t}$ represents all the heat gains and losses not modelled explicitly, which results from opening and closing doors, solar gains, and operation of other loads. The fourth constraint is (5), and assures that the interval of thermal confront $\left(\theta^{\text {Min }}-\theta^{\text {Max }}\right)$ defined by the consumer is satisfied, when the IAC is ON. The variable $\partial_{t}$ was included in constraint (5), in order to contemplate scenarios where the forecasted outdoor temperatures are below the minimum value of thermal comfort $\left(\theta^{\text {Min }}\right)$.

Figure 3 shows the behavior of an IAC used to cool a room with $40 \mathrm{~m}^{2}$. The characteristics of the IAC and of the room are described in Table I. The scheduling of the IAC took simultaneously into account all the temporal trajectories of forecasted outdoor temperatures presented in Figure 2 and the first temporal trajectory of forecasted electricity prices depicted in Figure 1. This scheduling also considered an interval of thermal comfort between 20 and $23{ }^{\circ} \mathrm{C}$. It was assumed the operation of the IAC during 24 hours.

The consumption of the IAC was defined to minimize the costs of buying electricity. Two main periods can be identified between 3:00-5:00 hours, where the EMS increases the consumption of the IAC to reduce costs. Other important aspect for the aggregator is the adoption of multiple scenarios of forecasted outdoor temperature. This approach reduces the uncertainty associated with the forecasts of IAC consumption, which consequently improves the bidding process of the aggregator.

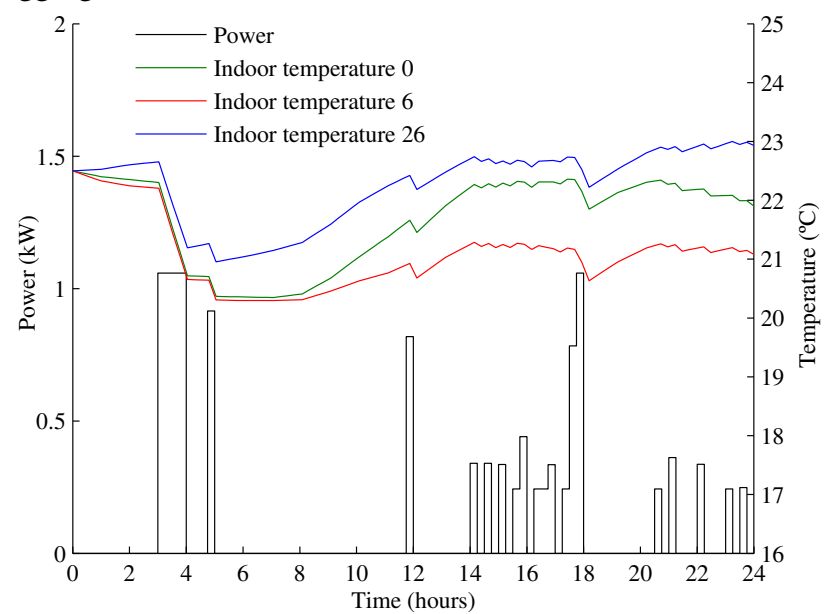

Figure 3. Behavior of the Inverter Air Conditioner

Regarding the refrigerator and the EWH, similar multitemporal optimization models can be used to schedule their operation. However, these appliances can only work in discrete mode (ON or OFF). So, to schedule the operation of the refrigerator, it is necessary to consider a different thermal equation. In this work, the following equation [5] was used.

$\theta_{t+1}=\varepsilon \theta_{t}+(1-\varepsilon)\left(\theta^{i}-\partial_{t} R P \eta\right)$ with $\varepsilon=e^{-\frac{\Delta t}{R C}}$

Instead of considering multiple temporal trajectories for the forecasted outdoor temperature as in (4), this optimization model only considers the indoor temperature $\left(\theta^{i}\right)$ of the room. In this model, the electric power $(P)$ of the refrigerator is a parameter, whereas $\partial_{t}$ is the only decision variable.

In case of the EWH, a similar thermal equation [5] to the one presented in (4) was used. However, instead of a resistance, it includes a heat loss constant $(\alpha)$. Additionally, the term $v_{t}$ is added to the model to represent the hot water consumption. The heat loss due to hot water consumption is calculated taking into account the difference between the desired temperature for hot water usage $\left(\theta^{d}\right)$ and the tank water inlet temperature $\left(\theta^{w}\right)$. The behavior of the EWH is set by the following thermal equation:

$$
\theta_{t+1}=\theta_{t}+\frac{\Delta t}{C}\left(-\alpha\left(\theta_{t}-\theta^{i}\right)-v_{t}\left(\theta^{d}-\theta^{w}\right)+\partial_{t} P\right)
$$

Concerning the $\mathrm{EV}$, its charging schedule is defined based on a scenario of price for two days ahead. The optimization model adopted for the EV scheduling is as follows [11]:

$\operatorname{Min} \sum_{t \in H_{y}^{F}}$ Price $_{j, t} P_{t} \Delta t$

\section{Subject to}

$$
\begin{aligned}
& 0 \leq P_{t} \leq P^{\operatorname{Max}}, \quad \forall t \in H_{y}^{F} \\
& \sum_{t \in H_{y}^{F}} \eta P_{t} \Delta t=C R_{y} \\
& y \in\{1, \ldots, N F\} ; \quad H_{y}^{F} \in\left\{t_{\text {initial }}, \ldots, t_{\text {final }}\right\}
\end{aligned}
$$


The objective function (9) consists in minimizing the cost of buying electrical energy in the day-ahead market for charging the EV. The model has two constraints. The first constraint (10) guarantees that the charging power of the EV $\left(P_{t}\right)$ is below the maximum charging power $\left(P^{\operatorname{Max}}\right)$. The second constrain (11) ensures that the charging requirements $(C R)$ of the end user are satisfied for each charging period $y$. The charging requirements are forecasted based on the desired state-of-charge and maximum available power [14]. Each charging period has an initial and final time instant $\left(t_{\text {initial }}\right.$ and $\left.t_{\text {final }}\right)$ and $N F$ is the number of charging periods. The parameter $H^{F}$ is the set of charging periods.

\section{E. Bidding in the Day-Ahead Spot Market}

The aggregator defines hourly bids for buying electrical energy in the day-ahead market, according to all forecasted consumption (flexible and inflexible) of type A and B clients.

Figure 4 illustrates the day-ahead spot market bids for August $9^{\text {th }}$ of 2010. Two types of bids are depicted: Flexible bids) all clients are type A; Inflexible bids) all clients are type B. As shown, with all clients as type $\mathrm{B}$, the consumption is placed in high price hours. On the other hand, with an optimized strategy, the bids related with the flexible loads are mostly placed in low price hours.

The results show that the adoption of a different scenario of forecasted electricity price by each client avoids the scheduling of all flexible loads in the same hour. As result, the flexible loads are distributed along the low price hours. This is a benefit for the aggregator since it generates bids with a higher quantity in low price periods. The data of this case study is detailed in Section IV.

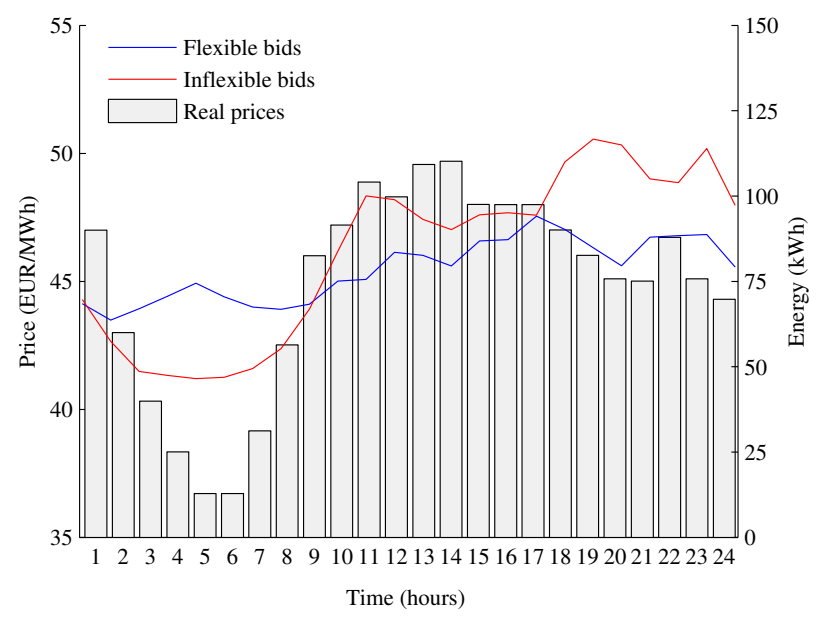

Figure 4. Distribution of Flexible versus Inflexible bids

\section{CASE STUDY}

\section{A. Description}

The models described in Section III were tested for an aggregator that has 200 residential and commercial clients under contract. Two scenarios were considered: Flexible biding) all clients are type A; Inflexible biding) all clients are type B. The consumers are from Porto (Portugal) and its aggregator participates in the Iberian electricity market. Seven summer days were considered (between $5^{\text {th }}$ and $11^{\text {th }}$
August 2010) to analyse and compare the economic impact of the Flexible versus Inflexible bidding.

The electricity consumption of type A clients was forecasted using simultaneously the optimization methods described in Section III and the load diagrams calculated by the Portuguese TSO [15]. Regarding the type B clients, their electricity consumption was forecasted using simultaneously the same load diagrams and the regular consumption of the flexible loads. Detail information about the models used to forecast the regular consumption can be found in [5] and [6].

The 200 consumers are characterized by 240 flexible loads composed by: 70 IAC, $40 \mathrm{EWH}, 100$ refrigerators and $30 \mathrm{EV}$. Table I presents the technical characteristics of two types of IAC and the physical characteristics of the rooms.

TABLE I. CHARACTERISTICS OF THE INVERTER AIR CONDITIONERS AND ROOMS

\begin{tabular}{cccccc}
\hline \hline $\begin{array}{c}\text { Room } \\
\text { area }\left(\mathrm{m}^{2}\right)\end{array}$ & $\begin{array}{c}P^{\text {Min }} \\
(\mathrm{kW})\end{array}$ & $\begin{array}{c}P^{\text {Max }} \\
(\mathrm{kW})\end{array}$ & $\eta$ & $\begin{array}{c}\mathrm{C} \\
\left(\mathrm{kWh} /{ }^{\circ} \mathrm{C} / \mathrm{m}^{2}\right)\end{array}$ & $\begin{array}{c}1 / R \\
\left(\mathrm{~kW} /{ }^{\circ} \mathrm{C} / \mathrm{m}^{2}\right)\end{array}$ \\
\hline $\begin{array}{c}{[25-30]} \\
{[30-35]}\end{array}$ & 0.19 & 0.84 & 3.82 & {$[0.02-0.065]$} & {$[0.002-0.003]$} \\
\hline $\begin{array}{c}{[35-40]} \\
{[40-45]}\end{array}$ & 0.24 & 1.06 & 3.78 & {$[0.02-0.065]$} & {$[0.002-0.003]$} \\
\hline \hline
\end{tabular}

The electricity consumption of the EWH is strongly dependent of the hot water demand for showers. The periods of showers were forecasted based on a probabilistic function for the shower occurrence [16]. Table II shows the characteristics of the EWH and the intervals of hot water demand considered. Table III shows the characteristics of the refrigerators. These characteristics were gathered from 5 different manufacturers.

TABLE II. CHARACTERISTICS OF THE ELECTRIC WATER HEATERS

\begin{tabular}{|c|c|c|c|c|c|c|}
\hline$P(k W)$ & $\begin{array}{c}C \\
\left(k W h /{ }^{\circ} \mathrm{C}\right)\end{array}$ & $\begin{array}{c}\alpha \\
\left(W /{ }^{\circ} \mathrm{C}\right) \\
\end{array}$ & $\theta^{d}\left({ }^{\circ} \mathrm{C}\right)$ & $\theta^{w}\left({ }^{\circ} \mathrm{C}\right)$ & $\theta^{i}\left({ }^{\circ} \mathrm{C}\right)$ & $\begin{array}{c}v \\
(l / \min ) \\
\end{array}$ \\
\hline 2 & 0.087 & 0.83 & [55-60] & 20 & 20 & {$[2-4]$} \\
\hline 2 & 0.116 & 0.97 & [55-60] & 20 & 20 & [2-4] \\
\hline 2.2 & 0.174 & 1.43 & [55-60] & 20 & 20 & {$[2-4]$} \\
\hline \multirow[t]{2}{*}{2.6} & 0.233 & 1.75 & {$[55-60]$} & 20 & 20 & {$[2-4]$} \\
\hline & TABLE III. & \multicolumn{5}{|c|}{ CHARACTERISTICS OF THE REFRIGERATORS } \\
\hline & $P(k W)$ & $C\left(k W h /{ }^{\circ} \mathrm{C}\right)$ & $R\left(k W /{ }^{\circ} \mathrm{C}\right)$ & $\eta$ & $\theta^{i}\left({ }^{\circ}\right.$ & \\
\hline & 0.08 & {$[0.03-0.07]$} & 76.67 & [3-4] & 20 & \\
\hline & 0.09 & [0.03-0.07] & 92.00 & [3-4] & 20 & \\
\hline & 0.10 & [0.03-0.07] & 107.33 & [3-4] & 20 & \\
\hline & 0.12 & [0.03-0.07] & 122.67 & [3-4] & 20 & \\
\hline
\end{tabular}

Regarding the EV charging, it was assumed a slow charging rate of $3.6 \mathrm{~kW}$ with overall efficiency of $90 \%$. The EV movement was simulated using a discrete-state, discretetime Markov chain described in [14]. The outputs of this method are the state-of-charge and the state (in movement or parked) of each EV. The charging requirements were forecasted based on the assumption that each $\mathrm{EV}$ owner requires the battery fully charged at the time of disconnection.

The temporal trajectories of electricity prices and outdoor temperatures were forecasted for the seven summer days previously referred. 


\section{B. Thermal comfort levels}

The interval of comfort for each thermal appliance varies with the consumer preferences. Therefore, for each case, the limits of the thermal comfort were randomly chosen. The maximum and minimum values assumed for the appliances were: IAC) between 20 and $26^{\circ} \mathrm{C}$; EWH) between 55 and 70 ${ }^{\circ} \mathrm{C}$; Refrigerator) between 3 and $8{ }^{\circ} \mathrm{C}$. These intervals were used to optimize the scheduling of the flexible loads (Flexible biding).

The regular consumption of the thermal loads was forecasted taking into account temperature set-points randomly chosen (Inflexible biding). The intervals of setpoints for the thermal appliance were: IAC) between 20 and $23^{\circ} \mathrm{C}$; EWH) 55 or $60^{\circ} \mathrm{C}$; Refrigerator) between 5 and $6{ }^{\circ} \mathrm{C}$.

\section{Results}

The average cost paid and the energy acquired by the aggregator in the day-ahead spot market were calculated for the seven days.

Figure 5 shows that flexible biding allows, simultaneously, reducing the average cost and the energy purchased by the aggregator in the market. In fact, the reduction of the global energy cost is greater (around 7\%) than the average cost per MWh, mostly due to the reduction of the energy bought. The reduction of the energy purchased is due to two reasons: 1) the adoption of thermal comfort intervals; 2) the forecasting of the EV charging is made for two days-ahead.

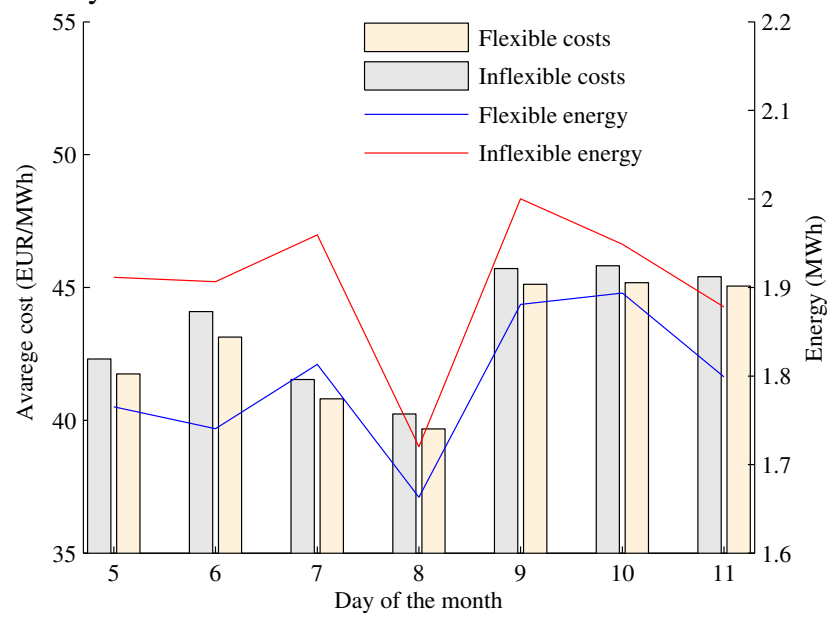

Figure 5. Evolution of the average cost paid and energy purchased along the seven days

\section{CONCLUSIONS}

A methodology to optimize aggregators' market bids was proposed in this paper, which schedules clients' flexible loads taking into account their preferences and temporal trajectories of forecasted outdoor temperatures and electricity prices. The methodology was tested using a case study with 200 residential and commercial consumers from the Iberian market.
It was found that the methodology proposed can lead to a significant increase of the aggregators' profit. For the case study addressed, a 7\% reduction in the cost of the energy purchased was achieved. The flexibility of the clients is thus a key factor to increase aggregators' profit in the day-ahead market. For this reason, aggregators should offer better retailing prices or discounts in the monthly bills of type A clients.

An important innovation introduced in this work was the consideration of multiple temporal trajectories (or scenarios) for outdoor temperatures and electricity prices forecasts. This approach hedges the risk of the aggregator, since the uncertainty associated with the electricity prices and the thermal loads consumption is greatly reduced.

\section{REFERENCES}

[1] P. Bertoldi, B. Hirl, N. Labanca, "Energy Efficiency Status Report 2012," JRC Scientific and Policy Reports, 2012.

[2] E. Hirst and B. Kirby, "Retail-Load Participation in Competitive Wholesale Electricity Markets," Edison Electric Institute Rep., Washington, D.C., 2001.

[3] R. N. Boisvert, P. A. Cappers, and B. Neenan, "The benefits of customer participation in wholesale electricity markets," Electricity J., vol. 15, no. 3, pp. 41-51, Apr. 2002.

[4] R. Belhomme et al., "ADDRESS technical and commercial conceptual architectures”, European Project ADDRESS, Deliverable 1.1., 2009.

[5] J. P. Iria, F.J. Soares, A. G. Madureira, M. Heleno, "Availability of household loads to participate in demand response," Power Engineering and Optimization Conference, 2014 IEEE 8th International, Mar. 2014.

[6] J. P. Iria, F.J. Soares, A. G. Madureira, "Advanced models and algorithms for demand participation in electricity markets," North American Power Symposium (NAPS), 2014, pp.1, 6, 7-9 Sept. 2014

[7] Z. Chen, L. Wu, Y. Fu, "Real-Time Price-Based Demand Response Management for Residential Appliances via Stochastic Optimization and Robust Optimization," Smart Grid, IEEE Transactions on, vol. 3, no. 4, pp. 1822-1831, Dec. 2012.

[8] A-H. Mohsenian-Rad, A. Leon-Garcia, "Optimal Residential Load Control With Price Prediction in Real-Time Electricity Pricing Environments," Smart Grid, IEEE Transactions on, vol.1, no.2, pp.120,133, Sept. 2010.

[9] E. Rivero et al., "Preliminary assessment of the future role of DSOs, future market architectures and regulatory frameworks for network integration of DRES," European Project EvolvDSO, Deliverable 1.3., Jul. 2014.

[10] http://www.wrf-model.org/index.php (accessed on March 2015)

[11] R.J. Bessa and M.A. Matos "Global against divided optimization for the participation of an EV aggregator in the day-ahead electricity market. Part I: theory," Electric Power Systems Research, vol. 95, pp. 309-318, Feb. 2013.

[12] R. Koenker and G. Bassett, "Regression quantiles", Econometrica, vol. 46, pp. 33-50, 1978

[13] M.C. Cario, B.L. Nelson, "Modeling and generating random vectors with arbitrary marginal distributions and correlation matrix" Technical Report, Department of Industrial Engineering and Management Sciences, Northwestern University, Evanston, IL, 1997.

[14] R.J. Bessa, M.A. Matos, F.J. Soares, J.A.P Lopes, "Optimized Bidding of a EV Aggregation Agent in the Electricity Market," Smart Grid, IEEE Transactions on, vol.3, no.1, pp.443,452, Mar. 2012.

[15] "Load profiles - 2010," Rede Eléctrica Nacional (REN), [Online] Available:http://www.mercado.ren.pt/PT/Electr/InfoMercado/Consumo /Paginas/PerfisConsumo.aspx

[16] M. Heleno, M.A. Matos, J.A.P Lopes, J.P. Iria, "Estimating the flexible residential load using appliances availability," Power Engineering and Optimization Conference, 2014 IEEE 8th International, Mar. 2014. 